\title{
Molecular Imaging Without Radiopharmaceuticals?
}

\author{
John C. Gore ${ }^{1-5}$, Thomas E. Yankeelov ${ }^{1-4,6}$, Todd. E. Peterson ${ }^{1-3}$, and Malcolm J. Avison ${ }^{1,2,7,8}$ \\ ${ }^{1}$ Institute of Imaging Science, Vanderbilt University, Nashville, Tennessee; ${ }^{2}$ Department of Radiology and Radiological Sciences, \\ Vanderbilt University, Nashville, Tennessee; ${ }^{3}$ Department of Physics and Astronomy, Vanderbilt University, Nashville, Tennessee; \\ ${ }^{4}$ Department of Biomedical Engineering, Vanderbilt University, Nashville, Tennessee; ${ }^{5}$ Department of Molecular Physiology and \\ Biophysics, Vanderbilt University, Nashville, Tennessee; ${ }^{6}$ Department of Cancer Biology, Vanderbilt University, Nashville, \\ Tennessee; ${ }^{7}$ Department of Neurology, Vanderbilt University, Nashville, Tennessee; and ${ }^{8}$ Department of Pharmacology, Vanderbilt \\ University, Nashville, Tennessee
}

\begin{abstract}
The limitations on the sensitivity for detecting small changes in $\mathrm{MRI}, \mathrm{CT}$, and ultrasound pulse-echo images are used to estimate the practical requirements for molecular imaging and targeted contrast enhancement for these modalities. These types of imaging are highly unlikely to approach the sensitivity for detecting molecular processes of radionuclear methods, and the prospects for achieving sufficient concentrations of appropriate agents in vivo are poor for several types of applications such as small-molecule targeting of specific receptors. However, using relatively large carrier systems such as particles and liposomes, sufficient concentrations of paramagnetic agents may be delivered to achieve MR-signal changes adequate for detection. The use of higher-resolution MR images will aid the prospects for molecular imaging in small animals. Theoretic considerations also predict that a similar approach, using rather large particles or carriers of materials with a high atomic number, may also be successful for CT, especially with additional developments such as the use of monochromatic x-rays. The prospects of molecular imaging by $\mathrm{x}$-ray imaging may not be as bleak as has been predicted. For ultrasound detection, gas-filled bubbles can provide a sufficient backscattered sound intensity to be detectable at concentrations and sizes not much different from agents designed for these other modalities.
\end{abstract}

Key Words: molecular imaging; MRI; x-ray CT; ultrasound

J Nucl Med 2009; 50:999-1007

DOI: 10.2967/jnumed.108.059576

\section{M} olecular imaging connotes "the visualization, characterization, and measurement of biologic processes at the molecular and cellular levels in humans and other living systems," and molecular imaging agents are "probes used to visualize, characterize, and measure biologic processes

Received Oct. 23, 2008; revision accepted Jan. 28, 2009.

For correspondence or reprints contact: John C. Gore, Vanderbilt University, Institute of Imaging Science, 1161 21st Ave. South, AA 1105 MCN, Nashville, TN 37232-2310.

E-mail: john.gore@vanderbilt.edu

COPYRIGHT @ 2009 by the Society of Nuclear Medicine, Inc. in living systems" (1). Recent developments in molecular biology and biochemistry, coupled with remarkable advances in imaging technologies, have accelerated progress in molecular imaging so that it has become a vital tool for basic research in preclinical studies, as well as increasingly important in clinical care as we move toward the era of personalized medicine. To date, most of the successful examples of molecular imaging have been derived from studies involving radionuclides and $\gamma$-ray imaging, or optical detection of fluorescent or bioluminescent molecules. By their natures and high sensitivities, PET and SPECT are clearly the primary methods available for translational molecular imaging in human subjects, and a relatively large number of imaging agents are potentially available for targeting specific biologic processes. Optical agents have thus far been substantially limited to smallanimal studies. Significant technical difficulties currently preclude the broader use of optical imaging in humans other than with invasive devices such as endoscopes, though there are continuing developments in diffuse optical tomography. Ultrasound imaging is in widespread clinical use, and intravascular microbubbles have been developed as contrast agents that may be labeled and selectively targeted to endothelial cell receptors, but the design and range of molecular imaging agents for use with ultrasound outside the circulation are clearly limited. The 2 most powerful radiologic imaging methods in routine clinical use are CT and MRI. These provide exquisitely detailed images of anatomy as well as various other types of information on tissue physiologic functions and composition. Recent developments in fast, multidetector CT and higher-field MRI with parallel imaging arrays have further enhanced the information available from these 2 modalities. There is therefore a natural enthusiasm for attempts to integrate the power of these imaging methods with evolving concepts in molecular imaging. Here, we review some of the physical limitations and potential 
opportunities for the likely success of such efforts, and for completeness we also consider limits on the sensitivity for detecting ultrasound contrast agents.

For several years, numerous attempts have been made to develop probes for specific targeting for detection by MRI, and these have enjoyed somewhat mixed success. There have also been some previous theoretic discussions of the likely limits to achieving molecular imaging with MRI (2-4), and these have highlighted the practical difficulties of achieving sufficiently high local concentrations of MRI contrast agents within tissue to be detectable. However, few reports have quantified the likely performance of molecular imaging with MRI in terms of theoretic limits from an imaging perspective. In addition, little consideration has been given to CT as a potential molecular imaging modality, and in general the possibilities of using x-ray methods for detecting targeted probes have been dismissed. Indeed, others have stated that $\mathrm{CT}$ "is not a molecular imaging technique per se" (5). Ultrasound is similarly not often considered as a modality suited for molecular imaging, and predicting the sensitivity for detecting ultrasound contrast agents is notoriously difficult because many factors that cannot be accurately estimated may affect sound scattering and detection. Here, we report the results of simple calculations of the requirements for successful detection of molecular processes using targeted agents for MRI, CT, and ultrasound pulse-echo imaging. Rather than consider specific biologic issues or targets, our approach starts by considering the known constraints on image quality that can be practically achieved and the limits of detection imposed by the physics of imaging. We present projectionsbased on simple calculations-showing that some common strategies for attempting molecular imaging with MRI are unlikely to be successful, that the sensitivity of CT could be comparable to MRI for some specific types of targeting, and that molecular imaging with either MRI or CT is theoretically achievable using particle-like carriers that can package and transport large numbers of atoms or molecules with appropriate properties. The absolute sensitivity for detecting particle-like agents of similar size by ultrasound is not hugely different. We hope these simple estimates will be of use for guiding possible developments of molecular imaging agents in each modality.

\section{MRI}

Two distinct approaches to detecting molecular imaging agents by MRI are possible. The first of these is direct detection of a nuclear species that is a component of the agent (e.g., nuclei of ${ }^{19} \mathrm{~F},{ }^{2} \mathrm{H}$, or ${ }^{13} \mathrm{C}$ within molecules, some of which may be hyperpolarized to increase sensitivity, such as helium and xenon gases $(6,7))$. The second approach is indirect detection via the effects of an agent on the large signal available from the hydrogen nuclei (protons) in tissue water. Regarding the second of these approaches, either of 2 methods is common: using paramagnetic or superparamag- netic agents that alter the tissue proton relaxation time $T_{1}, T_{2}$, or $T_{2}{ }^{*}$ (the basis of conventional MRI contrast agents (8)), or manipulating the magnitude of the water signal via specially designed radiofrequency irradiation that labels one species of protons (e.g., by selectively saturating the magnetization of protons within specific chemical groups) that in turn transfer the label to the water via magnetization exchange. This is the basis of so-called CEST (chemical exchange saturation transfer) and PARACEST (paramagnetic chemical exchange saturation transfer) agents (9).

The direct approach is the method most comparable to radionuclear imaging. However, detecting nuclei on the basis of magnetic induction is much less sensitive than counting emitted high-energy photons, especially when the magnetization is limited by the usual Boltzman distribution of energy states, whereby approximately only 1 in 100,000 of the nuclei contribute to the net signal. The most promising prospect for direct imaging without hyperpolarization is ${ }^{19} \mathrm{~F}$ (e.g., in perfluorocarbons). Fluorine has the largest gyromagnetic ratio after hydrogen and thus at a given field strength and concentration produces stronger signals than any other species. Because no background fluorine signal exists under normal physiologic conditions, detection of the presence of an agent requires merely that the measured signal be greater than the ambient level of noise, the random fluctuations in the apparent image background. However, unlike the cases for nuclear and x-ray imaging, the background noise variance in MRI is essentially independent of the signal (not counting "structured noise" such as motion effects, which can be signal-related) and is therefore the same magnitude for fluorine as for protons. Thus, the sensitivity for detecting small amounts of fluorine (by contrasting a small positive increase relative to the background noise) does not differ much from the sensitivity for detecting small variations in the large proton background caused, for example, by relaxation agents.

Multiple types of experiments with in vivo MRI/MR spectroscopy have established that in practice at common field strengths, the lower limit on the total amount of the detectable species is approximately $1 \mu \mathrm{mol}$ for detection with adequate signal-to-noise ratio (SNR) and in reasonable times (10). The necessary tissue concentration depends on the number and sizes of the imaging voxels. For example, using a typical volume coil to acquire the signal, for a wholebrain measurement (volume, $\sim 1 \mathrm{~L}$ ), approximately a $1 \mu \mathrm{M}$ concentration is detectable, whereas when measurements are made from a single, smaller voxel of volume $1 \mathrm{~mL}$, approximately $1 \mathrm{mM}$ is the required concentration. These are the approximate values found in practice; for example, for in vivo MR spectroscopy estimates of major metabolites such as $\mathrm{N}$-acetyl aspartate and choline, spectral images of dimension about $1 \mathrm{~mL}$ are typically acquired in reasonable times with an SNR of more than 30 (11). To achieve molecular imaging with, for example, fluorine at a spatial resolution of $2 \times 2 \times 5$ $\mathrm{mm}$, the volume-averaged concentration of fluorine atoms within the voxel must then be on the order of $50 \mathrm{mM}$ for the 
same SNR, and proportionately less $(\sim 5 \mathrm{mM})$ for mere detection above the noise.

For special cases and specific nuclei, a large increase $\left(\sim \times 10^{4}-10^{5}\right)$ in magnetization and thus MR signal can be achieved using hyperpolarization, by which the equilibrium Boltzman distribution is disturbed to achieve a much less even distribution of energy levels and, consequently, a larger magnetization (12). The hyperpolarization must take place out of the body by exposing appropriate substrates to some kind of spin-exchange mechanism, after which they may be introduced into the subject for imaging. This has been successfully achieved for the gases ${ }^{3} \mathrm{He}$ and ${ }^{129} \mathrm{Xe}$, and more recently for molecules containing ${ }^{13} \mathrm{C}$ or ${ }^{15} \mathrm{~N}$, allowing the exciting possibility of imaging metabolic pathways $(13,14)$. It may be noted that spectrally resolved imaging of such materials has considerable potential advantages over other imaging methods because high-resolution MR spectra reveal true chemical specificity: they are therefore arguably the most pure form of molecular imaging because the measured quantity changes with molecular chemistry. Nuclear imaging, by contrast, detects radionuclides but cannot distinguish between atoms in different molecules and thus is not sensitive to chemical changes. Hyperpolarization reduces the minimum required concentration by several orders of magnitude, allowing, for example, exquisite portrayal of the lungs (15) using direct imaging of helium in the airways at a resolution and SNR comparable to conventional proton imaging of water. Further work is needed, however, to establish the practical role of hyperpolarized labeled metabolites, especially given that in vivo the lifetime of the magnetization is usually quite limited (tens of seconds or less in many cases) and there may not be enough time for molecules to access sites of interest and be sufficiently involved in biochemical processes to provide adequate sensitivity. However, preliminary images have been produced depicting the uptake and turnover of substrates such as pyruvate and succinate $(16,17)$, and at the time of this review this approach to molecular imaging for quantifying the spatial distributions of metabolic fluxes appears promising.

Indirect detection of contrast agents remains the primary and most sensitive approach for molecular imaging with MRI. MRI contrast agents may affect relaxation times $T_{1}, T_{2}$, or $T_{2}{ }^{*}$, or they may be designed to modify the effects of magnetization transfer in the presence of off-resonance radiofrequency energy. More generally, their modes of operation may be classified into 4 different types for different applications: nonspecific contrast agents, such as the commonly used lanthanide chelates or intravascular blood pool agents, which do not incorporate any specific targeting strategies $(18,19)$; targeted contrast agents, which are usually paramagnetic species attached to or part of specifically engineered molecules, such as antibodies, which are directed toward and taken up by specific molecular targets (20,21); socalled smart contrast agents, which do not rely on selective targeting to achieve spatial specificity but instead change their efficacy (and thus their effects on the MR signal) only in response to specific local molecular characteristics (e.g., the presence of specific proteinases, or changes in environment such as $\mathrm{pH}$ [the ability to modulate the relaxivity of MRI contrast agents by physical or chemical factors within the tissue is a unique feature and potential advantage of MRI agents $(22,23)]$ ); and labeled cells, by which agents can be bound to or introduced into specific cell types, which then rely on the trafficking and recognition of the cells for their localization $(24,25)$.

Just as the sensitivity for detecting the presence of radionuclei depends on the SNR, our ability to detect relaxation effects also depends on the magnitude of the effect of the agent compared with a background relaxation rate; that is, the magnitude of changes due to the agent must be significantly different from the intrinsic relaxation rate within tissues. An upper limit on relaxation times is given by pure water, in which dipole-dipole interactions between protons shorten $T_{1}$ to approximately $4 \mathrm{~s}$. For most soft tissues at clinical imaging fields, the longitudinal relaxation rate $R_{1}\left(\equiv 1 / T_{1}\right)$ is higher, approximately $1 \mathrm{~s}^{-1}$. Given the intrinsic variations within a tissue (a few percentage points) and the available SNR of typical images, it is reasonable to stipulate the minimum detectable change in background relaxation rate to be on the order of $10 \%$; that is, we require the minimum change in rate caused by an agent, $\Delta R_{1 \mathrm{~min}}$, to be approximately $0.10 \mathrm{~s}^{-1}$. The efficacy of relaxation agents in MRI is usually described in terms of the relaxivity, or the change in $R_{1}$ per unit concentration of agent. The most common agents in use, such as gadolinium-DTPA, have relaxivities of approximately $4 \mathrm{~s}^{-1} \mathrm{mM}^{-1}$ (slightly more or less at lower or higher field strengths, respectively), but in principle the relaxivity of some paramagnetic agents may be enhanced by, for example, binding to a macromolecule (a discussion of the factors affecting MRI contrast agents has been previously published (26)). If the enhancement factor relative to gadoliniumDTPA is $\epsilon$, then the relaxivity of the agent is $4 \epsilon \mathrm{s}^{-1} \mathrm{mM}^{-1}$; setting $\Delta R_{1 \text { min }}$ equal to $0.10 \mathrm{~s}^{-1}$, the necessary concentration for detection is

$$
C=\frac{\Delta R_{1 \mathrm{~min}}}{4 \varepsilon \mathrm{s}^{-1} \mathrm{mM}^{-1}}=\frac{25}{\varepsilon} \mu \mathrm{M}, \quad \text { Eq. } 1
$$

where $C$ is the $\mu \mathrm{M}$ concentration of the contrast agent.

The Solomon-Bloembergen-Morgan equations (26) describe how relaxivity varies with factors such as the magnetic field, the correlation times of the local fluctuating fields that promote relaxation, and the distance between the paramagnetic species and the relaxing nuclei; in theory, enhancements of roughly 2 orders of magnitude may be possible. To date, however, these have not been achieved, and in practice only more modest gains may be possible in vivo. Thus, if a reasonable achievable value for $\epsilon$ is taken to be 25 , our required concentration of metal (gadolinium) is $1 \mu \mathrm{M}$. This is much higher than the levels detected in, for example, nuclear imaging methods such as PET and represents 2 facts. Not only is MRI fundamentally an insensitive technique (it may not seem insensitive given the high quality of MR 
images, but recall that these are acquired by detecting water protons that are roughly $80 \mathrm{M}$ in tissues), but also there is already a rather high level of background relaxation in tissue against which the agent must contrast, whereas in nuclear imaging there is no background.

To put this concept into perspective, assume that tissues contain about $10^{12}$ cells per liter (each approximately 1,000 $\mu \mathrm{m}^{3}$ ) and that the concentration of agent needed for detection is therefore equivalent to $10^{-18} \mathrm{~mol}$ per cell, with all cells in the voxel or region of interest labeled. It is necessary, therefore, to deliver something on the order of $10^{6}$ atoms of the paramagnetic species (e.g., gadolinium atoms) to every cell in the region of enhancement. For small-molecule targeting with one or a few metal ions per molecule, $10^{6}$ would have to be the number of ligand sites or receptors in each cell that bind the agent.

Alternately, these atoms might be delivered within a single particle or package such as a liposome, or attached in large quantities to a dendrimer or biopolymer. The relaxation effects of the paramagnetic payload may then be spread to affect all other water within the voxel as long as the water is free to exchange in and out of the vicinity of the metal ions in the time scale available. For example, if liposomes contain a solution that is $1 \mathrm{M}$, then a single targeted liposome of diameter $100 \mathrm{~nm}$ in every cell in the target region would produce the required contrast (i.e., a concentration of $1 \mu \mathrm{M}$ of gadolinium). If only one cell in a thousand takes up a particle, then the liposome must be $1 \mu \mathrm{m}$ to achieve the same enhancement. The important quantity is the average concentration of metal within the voxel; if the agent is highly localized, then it may be advantageous to acquire images with higher spatial resolution to minimize dilution of the effect by partial-volume averaging. In small-animal and preclinical studies, voxels may be reduced to approximately $100 \mu \mathrm{m}$ in each dimension. Table 1 summarizes the numbers and sizes of such targeted containers that are then required to meet the minimal levels to affect the signal significantly. For voxels of $1 \mathrm{~mm}$, the numbers must be $\times 1,000$. For concentrations less than $1 \mathrm{M}$, the numbers scale in inverse proportion. For large particles, it may be possible to make a $T_{1}$ agent for single-cell imaging or tracking at high spatial resolution. However, even if the membrane separating the agent from tissue water is highly permeable and allows free exchange

\begin{tabular}{|c|c|c|}
\hline $\begin{array}{l}\text { Diameter of } \\
\text { particle containing } \\
1 \mathrm{M} \text { gadolinium } \\
\text { solution }(\mathrm{nm})\end{array}$ & $\begin{array}{l}\text { [Gadolinium] in } \\
\text { tissue }(\mu \mathrm{M}) \text { if } \\
1 \text { particle per } \\
(100 \mu \mathrm{m})^{3} \text { voxel }\end{array}$ & $\begin{array}{l}\text { No. of particles } \\
\text { per cell to achieve } \\
1 \mu \mathrm{M} \text { to produce } \\
\Delta \mathrm{R}_{1} \sim 0.1 \mathrm{~s}^{-1} \\
\text { in }(100 \mu \mathrm{m})^{3} \text { voxel }\end{array}$ \\
\hline 10 & $0.5 \times 10^{-6}$ & 2,000 every cell \\
\hline 100 & $0.5 \times 10^{-3}$ & 2 every cell \\
\hline 1,000 & 0.5 & 1 every 500 cells \\
\hline
\end{tabular}

of water, the effectiveness of single particles is limited. Free water at body temperature has a self-diffusion rate $D$ of approximately $3 \times 10^{-5} \mathrm{~cm}^{2} \mathrm{~s}^{-1}$, but in tissues the effective diffusion coefficient is lower because of restrictions and is typically $\sim 1 \times 10^{-5} \mathrm{~cm}^{2} \mathrm{~s}^{-1}$. Using the Einstein relationship for diffusion, the mean displacement of a water molecule $\langle l\rangle$ in a time $t=1 \mathrm{~s}\left(\sim \mathrm{T}_{1}\right)$ is then only $\langle l\rangle=\sqrt{2 D t} \approx 45 \mu \mathrm{m}$. Thus, on average a volume of only that radius is effectively relaxed, and water farther away will not be affected by the paramagnetic agent.

An alternative to $T_{1}$-reducing paramagnetic agents is provided by so-called susceptibility agents, of which superparamagnetic iron oxide particles are a prime example (27). These reduce tissue transverse relaxation rates, especially $1 /$ $T_{2}{ }^{*}$, and do not rely to the same extent on the exchange of water to spread their effects. By altering the magnetic field in their vicinity, iron oxide particles induce signal dephasing within the nonuniform field and their effects extend over distances that depend on their size and other factors. A simple iron oxide such as $\mathrm{Fe}_{2} \mathrm{O}_{3}$ has a density on the order of 5, and therefore within each particle the effective molarity of iron is about 60 . Moreover, the relaxivity per iron atom can be quite high, such that the enhancement factor can be approximately 100 (corresponding to a relaxivity of $400 \mathrm{~s}^{-1} \mathrm{mM}^{-1}$ iron). However, the background relaxation rate that must be altered is much higher than $R_{1}$-typically, $R_{2} *$ is about $20 \times R_{1}$. Thus, instead of a change in $R_{1}$ of 0.1 we seek to achieve a change in $R_{2} *$ of 2 . Roughly, therefore, the relative efficacy of $T_{2}{ }^{*}$ agents versus $T_{1}$ agents can be about $60 \times 4 / 20=12$ times greater than particles containing $1 \mathrm{M}$ gadolinium. Table 2 shows the estimated numbers of particles of different sizes required to achieve significant effects. For larger particles, it should be possible to detect a single particle in a small voxel.

\section{CHEMICAL EXCHANGE SATURATION TRANSFER AGENTS}

An alternative class of contrast agents has been developed in recent years. These agents use magnetization transfer effects between water molecules in the tissue and protons in an exogenous agent. The protons have a different resonance frequency (a chemical shift) and usually undergo chemical exchange at tissue $\mathrm{pH}$. Contrast effects are manipulated by applying radiofrequency pulses at the resonance frequency of these labile protons - such as amide protons-which changes their magnetization (they become completely or partially saturated). This saturation is then communicated to the water by magnetization transfer, mainly through chemical exchange, so that the larger bulk water signal is decreased, thereby amplifying the presence of the labile species. This is the basis of CEST and PARACEST agents (which incorporate a paramagnetic center to shift the resonance frequencies farther apart) $(28,29)$. The signal change depends on a constant, $k$, that denotes the rate of exchange between the different species. CEST effects may be quite sensitive to physicochemical factors such as $\mathrm{pH}$. In addition, because they are present 
TABLE 2. Requirements for $T_{2}$ Agents in MRI: Number of Particles Required Scales Inversely with Relaxivity of Carrier Particle

\begin{tabular}{|c|c|c|}
\hline $\begin{array}{l}\text { Diameter of particle } \\
\text { containing iron } \\
\text { oxide }(\mathrm{nm})\end{array}$ & $\begin{array}{c}\text { [Iron] in tissue } \\
(\mu \mathrm{M}) \text { if } 1 \text { particle } \\
\text { per }(100 \mu \mathrm{m})^{3} \\
\text { voxel }\end{array}$ & $\begin{array}{l}\text { No. of particles } \\
\text { per cell to achieve } \\
5 \mu \mathrm{M} \text { iron to } \\
\text { produce } \Delta \mathrm{R}_{2} \sim 2 \\
\text { in }(100 \mu \mathrm{m})^{3} \text { voxel }\end{array}$ \\
\hline 10 & $30 \times 10^{-6}$ & 166 every cell \\
\hline 100 & $30 \times 10^{-3}$ & 1 every 6 cells \\
\hline 1,000 & 30 & 1 every 6,000 cells \\
\hline
\end{tabular}

only when the radiofrequency pulse is applied, they can be switched on and off at will. Tissue itself is approximately $80 \mathrm{M}$ in water protons (pure water is $110 \mathrm{M}$ ). If particles (e.g., dendrimers populated with exchanging protons) are designed as CEST agents, then a practical aim would be to achieve a $10 \%$ reduction in the water signal in a voxel on applying the radiofrequency pulse because of the presence of the agent. The change in the water signal will be larger for high $k$ values, though these cannot be too high or else the resonances are no longer separable and the effect cannot be measured. Because exchange rates on the order of $10^{4}$ $\mathrm{s}^{-1}$ are quite possible, the effective molarity of the exchanging protons need be approximately only $10^{-5}$ that of water, or $0.8 \mathrm{mM}$. Particulate agents may be populated with multiple surface groups and exchangeable sites, whereas carriers such as liposomes can accommodate high molar concentrations of exchangeable protons. Table 3 shows the estimated numbers of appropriately designed carriers required to be detectable. The choice of a high exchange rate places significant constraints on both the chemical shift of the exchanging species (it needs to be much greater than $\sim 30 \mathrm{ppm}$ even at $7 \mathrm{~T}$ for the difference in resonance frequencies to be $>>10^{4} \mathrm{~s}^{-1}$ ) and on the free exchange of water through any membranes.

\section{CT}

To consider and compare the potential for CT agents, we first discuss some relevant aspects of the physics of CT and consider the tradeoffs of radiation dose, spatial resolution, and contrast sensitivity. The reduction in the intensity of a monochromatic X-ray beam as it travels through tissue may be described in terms of the total linear attenuation coef- ficient, $\mu$, usually expressed in units of $\mathrm{cm}^{-1}$. This coefficient represents the fraction of the beam lost per unit distance of penetration into the medium. In the diagnostic range exploited by CT, $\mu$ is dominated by 2 types of photon-tissue interactions, the Compton and photoelectric effects. The photoelectric and Compton interactions vary in different ways with the energy of the x-ray beam and the composition of the material. They contribute approximately equally to soft-tissue absorption at $25 \mathrm{keV}$, but as the photon energy $(E)$ increases, the probability of photoelectric interaction decreases rapidly until, by $100 \mathrm{keV}$, the Compton effect contributes nearly $100 \%$ to the total attenuation. The probability of a photoelectric interaction is proportional to the cube of the atomic number, $Z^{3}$, of the attenuating medium and decreases continuously as approximately $1 / E^{3}$ except for discontinuities around the binding energies of electrons within the absorber; when the photon energy is just higher than an electron shell binding energy, the probability of a photoelectric interaction increases by a significant factor (30) and these edge phenomena can be important in selecting for higher- $Z$ materials as contrast agents. The probability of a Compton interaction is almost independent of $Z$ but is proportional to the electron density of the attenuating medium, which is relatively uniform for many materials. For comparing material properties, it is useful to consider the mass attenuation coefficients of materials, $\mu / \rho$, which are simply the total linear attenuation coefficients divided by the density of the material. Table 4 lists the mass attenuation coefficients $\mu / \rho$ for several atoms of interest at relevant photon energies.

CT scanners use polyenergetic X-ray beams but record distributions of the x-ray total linear attenuation coefficient at an effective energy and display them in terms of Hounsfield units, HU, which are normalized values relative to water:

$$
\mathrm{HU}=1,000\left(\frac{\mu-\mu_{\text {water }}}{\mu_{\text {water }}}\right),
$$

where $\mu$ is the linear attenuation coefficient of the tissue and $\mu_{\text {water }}$ is the linear attenuation coefficient of water. To be detectable using CT, a contrast agent must change the attenuation coefficient within one or more voxels to an appreciable extent. The ability to detect such a change is a function of the radiation dose and spatial resolution, which

\begin{tabular}{|c|c|c|c|c|}
\hline $\begin{array}{c}\text { Diameter of particle containing } \\
\text { exchangeable protons }(\mathrm{nm})\end{array}$ & $\begin{array}{l}\text { No. of exchangeable } \\
\text { protons }\left(k=10^{4} \mathrm{~s}^{-1}\right) \\
\text { required per particle }\end{array}$ & $\begin{array}{l}\text { Required molarity of } \\
\text { exchangeable protons } \\
\text { within particle }\end{array}$ & $\begin{array}{l}\text { Particles per } \\
(100 \mu \mathrm{m})^{3} \\
\text { voxel }\end{array}$ & $\begin{array}{c}\text { Particles per cell } \\
\text { within }(100 \mu \mathrm{m})^{3} \\
\text { voxel }\end{array}$ \\
\hline 100 & $4.8 \times 10^{5}$ & $750 \mathrm{mM}$ & $10^{6}$ & 1,000 \\
\hline 1,000 & $4.8 \times 10^{5}$ & $750 \mu \mathrm{M}$ & $10^{6}$ & 1,000 \\
\hline 1,000 & $4.8 \times 10^{8}$ & $750 \mathrm{mM}$ & $10^{3}$ & 1 \\
\hline 10,000 & $4.8 \times 10^{5}$ & $0.75 \mu \mathrm{M}$ & $10^{6}$ & 1,000 \\
\hline 10,000 & $4.8 \times 10^{8}$ & $750 \mu \mathrm{M}$ & $10^{3}$ & 1 \\
\hline 10,000 & $4.8 \times 10^{11}$ & $750 \mathrm{mM}$ & 1 & 0.001 \\
\hline
\end{tabular}


TABLE 4. X-Ray Properties for Various (Relevant) Materials with K- or L-Edges in the Diagnostic Energy Range

\begin{tabular}{|c|c|c|c|}
\hline Material & $\mu / \rho$ at $30 \mathrm{keV} \mathrm{cm}^{2} / \mathrm{g}$ & $\mu / \rho$ at $60 \mathrm{keV} \mathrm{cm}^{2} / \mathrm{g}$ & $\mu / \rho \mathrm{cm}^{2} / \mathrm{g}$ at $\mathrm{K}$ or L edge (keV) \\
\hline Tissue (water) & 0.38 & 0.20 & NA \\
\hline Iron & 8.18 & 1.21 & $407.6(7.11)$ \\
\hline lodine & 8.56 & 7.58 & $35.8(33.2)$ \\
\hline Barium & 9.90 & 8.51 & 28.6 (37.4) \\
\hline Gadolinium & 14.84 & 11.75 & $18.6(50.2)$ \\
\hline Lead & 30.32 & 5.02 & $154.8(15.9)$ \\
\hline
\end{tabular}

in turn determine the SNR within the image. CT relies on detecting photons, and just like nuclear imaging, the statistical variability of the measurements used to reconstruct images is limited by the number of photons contributing to each measurement. This statistical limit is set by the radiation dose (which limits the total number of photons contributing) and the spatial sampling (or resolution). The SNR measures the random variation from voxel to voxel in estimated values of $\mu$ that are apparent even for a uniform region. Any real change in attenuation must exceed these random background variations by a minimum factor (typically 3-5 according to the size and shape of the feature). These quantities are related in a well-established manner (31):

$$
\mathrm{SNR}^{2}=\left(\frac{\bar{\mu}}{\sigma}\right)^{2} \propto a^{3} D t
$$

where $a$ is the in-plane spatial resolution, $t$ is slice thickness, $D$ is radiation-absorbed dose, and $\sigma$ represents the SD of the estimated mean value $\bar{\mu}$. On the basis of a simple model of detection, the minimum change in attenuation, $\Delta \mu_{\min }$, that can be detected is

$$
\frac{\Delta \mu_{\min }}{\bar{\mu}} \propto \frac{1}{\sqrt{a^{3} t D}} .
$$

Note that there is a considerable penalty in SNR when small voxels are chosen and that the contrast sensitivity can be dramatically improved by using a lower resolution, as can be achieved simply by averaging voxels after acquisition. From experience with clinical scanners, for an isotropic spatial resolution of approximately $1 \mathrm{~mm}$ and doses on the order of $0.01-0.02 \mathrm{~Gy}$, the contrast detection limit is approximately a $1 \%$ difference in $\bar{\mu}$ (32). Studies using dedicated small-animal scanners typically use higher doses and much smaller voxels.

We can now consider how much of a potential particlelike molecular imaging contrast agent will be required within an imaging voxel to induce a detectable change. We can couch the estimate in terms of what volume fraction, $V_{f}$, within an imaging voxel or region must be occupied to generate a large enough change in attenuation. The measured linear attenuation coefficient for a voxel due to the presence of a contrast agent that occupies a volume fraction $V_{f}$ is given by

$$
\left(\mu_{t}+\Delta \mu\right)=\left(1-V_{f}\right) \mu_{t}+V_{f} \mu_{a}
$$

where $\mu_{t}$ and $\mu_{a}$ are the linear attenuation coefficients of the tissue and contrast agent, respectively, and $\Delta \mu$ is the change in attenuation caused by the agent. By taking $\mu_{t}<$ $<\mu_{a}$, we see that $\Delta \mu=V_{f} \mu_{a}$; from Equation 4 , the necessary volume fraction for the agent to be detected is

$$
V_{f} \propto \frac{1}{\sqrt{a^{3} t D}} \frac{\mu_{t}}{\mu_{a}} .
$$

If the volume fraction is occupied as several identical (spheric) particles of diameter $d$, we can calculate the number per volume, $N$, to achieve the required result. Replacing the linear attenuation coefficients with mass attenuation coefficients, we obtain

$$
N \propto \frac{6}{\pi d^{3}} \frac{(\mu / \rho)_{t} \cdot \rho_{t}}{(\mu / \rho)_{a} \cdot \rho_{a}} \frac{1}{\sqrt{a^{3} t D}}=\frac{6}{\pi d^{3}} \frac{(\mu / \rho)_{t} \cdot \rho_{t}}{(\mu / \rho)_{a} \cdot \rho_{a}} \frac{\Delta \mu_{\min }}{\bar{\mu}}
$$

where $\rho_{t}$ and $\rho_{a}$, are the densities of soft tissue and the contrast agent, respectively.

If we assume we can measure a $1 \%$ change in $\mu_{t}$ by operating at clinical doses and resolution $(a, t \sim 1 \mathrm{~mm}, D \sim$ $0.01 \mathrm{~Gy}$ ) and take the density of soft tissue to be approximately $1 \mathrm{~g} / \mathrm{cm}^{3}$, we can calculate $N$ for different sizes and compositions of particles. These data are summarized in Table 5. We take the mass attenuation coefficient of tissue to be $0.2 \mathrm{~cm}^{2} / \mathrm{g}$ at $60 \mathrm{keV}$ and $0.38 \mathrm{~cm}^{2} / \mathrm{g}$ at $30 \mathrm{keV} \mathrm{(33)} \mathrm{and}$ consider the necessary sizes and numbers of particles composed of 3 pure metals: iron, gadolinium, and lead.

The sensitivity for relatively large metal particles is comparable to that predicted for MRI contrast agents and should be compared with Tables 1-3 above. Table 5 shows the predicted requirements for different-sized particles to achieve a $1 \%$ contrast in a $1-\mathrm{mm}^{3}$ voxel at $0.01 \mathrm{~Gy}$.

Several potential methods exist to improve the sensitivity of CT for molecular imaging, particularly in preclinical studies. At present, these methods are not being explored, 


\begin{tabular}{|c|c|c|c|}
\hline $\begin{array}{l}\text { Diameter of particle } \\
\text { containing metal }(\mathrm{nm})\end{array}$ & $\begin{array}{l}\left.\frac{\left(\mu / \rho_{1}\right)_{1}}{(\mu / \rho)}\right)_{a} \cdot p_{a} \\
\text { specific } k e V\end{array}$ & $\begin{array}{l}\text { Particles per } \mathrm{cm}^{3} \\
\text { to be detected }\end{array}$ & $\begin{array}{c}\text { Particles per cell to be } \\
\text { detected }\end{array}$ \\
\hline 100 & 0.002 for gadolinium at $60 \mathrm{keV}$ & $4 \times 10^{10}$ & 40 per cell \\
\hline 1,000 & 0.002 for gadolinium at $60 \mathrm{keV}$ & $4 \times 10^{7}$ & 1 every 25 cells \\
\hline 10,000 & 0.002 for gadolinium at $60 \mathrm{keV}$ & $4 \times 10^{4}$ & 1 every 25,000 cells \\
\hline 100 & 0.006 for iron at $30 \mathrm{keV}$ & $12 \times 10^{10}$ & 120 per cell \\
\hline 1,000 & 0.006 for iron at $30 \mathrm{keV}$ & $12 \times 10^{7}$ & 1 every 8 cells \\
\hline 10,000 & 0.006 for iron at $30 \mathrm{keV}$ & $12 \times 10^{4}$ & 1 every 8,000 cells \\
\hline 100 & 0.001 for lead at $30 \mathrm{keV}$ & $2 \times 10^{10}$ & 20 per cell \\
\hline 1,000 & 0.001 for lead at $30 \mathrm{keV}$ & $2 \times 10^{7}$ & 1 every 50 \\
\hline 10,000 & 0.001 for lead at $30 \mathrm{keV}$ & $2 \times 10^{4}$ & 1 every 50,000 cells \\
\hline
\end{tabular}

and the performance limits of commercial scanners have been set to achieve other ends that are not easily compatible with molecular imaging needs. For example, clinical scanners use relatively high resolution and the contrast sensitivity is limited by the dose, which itself is limited by patient-exposure considerations. However, Equation 4 shows how a modest decrease in resolution would lead to dramatic increases in contrast sensitivity. PET and SPECT are performed with a resolution 5-10 times worse than that of CT. Thus, we ought to evaluate the potential for CT using comparable parameters; that is, envision the use of human CT with degraded spatial resolution. We see, for example, that increasing the dose by 2 and degrading the in-plane resolution by 4 and the thickness by 2 would reduce by 16 -fold the number of particles per volume required. In small animals, if we sacrifice the exquisite resolution commonly obtained (routinely better than 100 $\mu \mathrm{m})$ and operate with the spatial resolution of small-animal PET ( $\sim 1 \mathrm{~mm}$ ), we need on the order of one 1,000-nm lead particle every 200 cells at a dose of $0.16 \mathrm{~Gy}$. This is quite comparable with the best obtainable with MRI albeit at lower resolution.

Another strategy for increasing the sensitivity to targeted CT contrast agents would be to exploit the existence of $\mathrm{K}$-edges in the agent of interest or (more powerfully) to use quasimonochromatic $\mathrm{x}$-rays or energy-subtraction strategies $(34,35)$. Exploiting the K-edge appropriately, by comparing images from $\mathrm{x}$-ray beams whose dominant photons have energies just above and below the K-edge, or using monochromatic beams at the energy of the K-edge, could enhance sensitivity by another order of magnitude, based on the values of absorption in Table 4, even after one considers the increase in background noise that accompanies subtraction imaging at constant dose.

\section{ULTRASOUND PULSE-ECHO IMAGING}

Estimating the sensitivity for detecting targeted contrast agents by ultrasound pulse-echo methods is complex because several factors that are important are not known or cannot be easily generalized. Nonetheless, we can consider a simple model of detecting ultrasound contrast agents to make some reasonable comparison to other modalities. For example, irrespective of the means of directing agents to specific targets, a feasible and sensitive source of ultrasound contrast comes from the use of small bubbles filled with a gas whose density and elasticity are very different from tissues so that they behave as almost perfect scatterers or reflectors of incident sound. The effective cross-section for backscattering will depend on the type of bubbles, their sizes, and their precise arrangement within tissue. Multiple small bubbles within a volume can give rise to interference effects, and multiple bubbles may scatter coherently or incoherently (36), but a best-case scenario occurs when the sound waves scattered by multiple bubbles add constructively in linear fashion.

To be sure that backscattered echoes from bubbles are detected and recognized as having been produced by the contrast agent, the backscattered signal must be significantly greater than any other echo source at the same location or any other source of spurious detector or receiver noise that might be placed at that location in the image. The latter sources of noise correspond, in the limit of performance, to Johnson-type noise variations in the front end of the receiver amplifier or transducer but, with improvements in technology, such thermal noise sources can be made negligible compared with other types of image variance. More important, ultrasound pulse-echo techniques are sensitive to variations within a medium that give rise to small-scale backscattered echoes even from within a macroscopically uniform tissue and even without discrete inhomogeneities. Basic acoustic theory predicts that ultrasound speckle will arise whenever spatial variations exist in the fundamental acoustic properties of density or compressibility (36). If the density and elastic properties are spatially variant, an incident sound pressure wave will be partially scattered into different directions, including back toward the source. Gore and Leeman (37) showed that the relevant intrinsic spatial variations in acoustic properties (the fractional deviations of the local density and compressibility relative to their means) that give rise to scattering are effectively smoothed by the emitted sound pulse used; thus, the scattering volume to be considered is the instantaneous, pressure amplitudeweighted, volume of the pulse. For example, most imaging in humans occurs at frequencies of $5 \mathrm{MHz}$ or below. From diffraction theory, the lateral extent of the sound field 
produced by an ultrasound transducer (the beam width) depends on the ratio of the size of the transmitter aperture to the wavelength, though this is modified when focusing is used. The beam width can be described, for example, as the distance off the transducer axis at which the pressure has fallen to half its axial (maximum) value and is typically about 5 wavelengths, whereas the pulse length is typically only $2-3$ wavelengths for high-resolution imaging systems. In practice then, for practical pulsed-sound fields, an estimate of the volume of tissue occupied at any instant by a single sound pulse is approximately $40 \lambda^{3}$, where $\lambda$ is the wavelength $(300 \mu \mathrm{m}$ in tissue at $5 \mathrm{MHz}$, where the speed of sound is $1,500 \mathrm{~m} \mathrm{~s}^{-1}$ ). Thus, the volume from which scattering occurs at any time is approximately $10^{9} \mu^{3}$, or $1 \mathrm{~mm}^{3}$, corresponding to $10^{6}$ cells.

Our ability to detect one or more contrast agent bubbles within such a volume (which also corresponds to the resolution element of the ultrasound image) will depend on whether the backscattered sound exceeds any low-level scattering from within this volume arising from fluctuations in the tissue acoustic properties. It is difficult to estimate in general how small such fluctuations may be, especially when other tissue structural heterogeneities are also present. However, a best-case scenario would arise when the only significant variations are from the inherent stochastic nature of tissue composition. The mean density and compressibility of tissue may be expected to mirror the number of cells per volume, and we can hypothesize that the fractional deviation in acoustic properties (which determines the scattering cross section (37)) is then proportional to the fractional variation in cell density. For example, within a volume $V$ we expect on average to find several cells $N$, each of volume $v$ and density $\rho_{c}$. There will also be a corresponding number of extracellular spaces, each of volume $u$ and density $\rho_{e}$. The overall density of this volume is then

$$
\rho=\frac{N v \rho_{c}+N u \rho_{e}}{V} .
$$

The density of any other similar volume will be slightly different because $N$ varies randomly in space. Thus, if the mean density is $\bar{\rho}$, which corresponds to a mean cell density of $\bar{N}$, then the fractional deviation in density is given by

$$
\frac{\rho-\bar{\rho}}{\bar{\rho}}=\frac{N-\bar{N}}{\bar{N}} .
$$

A similar argument can be applied to fluctuations of compressibility, which also give rise to scattering (37). The statistical distribution of cell number density in different volumes will resemble a Poisson distribution, so that the statistical variance in number of cells $N$ can be approximated by the mean, $\bar{N}$. Thus, if the pulse volume $V$ on average contains $10^{6}$ cells, the SD is expected to be $10^{3}$ and the average fractional deviation, compared with the mean, is $10^{-3}$. Thus, even in the absence of obvious structural inhomogeneities, about $0.1 \%$ of the sound amplitude is expected to be backscattered from within cellular tissues. From this we may estimate how large a bubble (of radius $R$ ) or how many bubbles $(n)$ must be contained within the volume of the pulse to produce scattering larger than this amount. If each bubble behaves as a small-volume scatterer, and the echo from each bubble adds coherently (36), then the total volume occupied by bubbles within the pulse volume, $V$, must satisfy

$$
V=\frac{4}{3} \pi n R^{3} \gg 10^{9} \times 10^{-3}=10^{6} \mu \mathrm{m}^{3} .
$$

Table 6 shows values of $R, n$, and the number of bubbles per cell, to be detected. For bubbles directed toward vascular targets, the volume of distribution is restricted to the intravascular fraction of tissue, which is only a few percentage points of the total. For example, if the vascular volume is $x \%$ of tissue, we require $\frac{10}{x} \%$ of the blood to be replaced by bubbles in the target volume of interest.

\section{DISCUSSION}

The presented analyses are not intended to be exact calculations of the amounts of contrast required for any specific application. Success in detecting a molecular imaging probe will always depend on a host of factors that are not easily quantified in advance of an experimental study, but these simple order-of-magnitude estimates can be used as guides to assess the likelihood of success of particular strategies. Realistically, most molecular imaging in the foreseeable future will remain the domain of nuclear imaging, though in animals there will be a large role for optical methods too. CT and MRI will continue to provide exquisite anatomic images that may be overlaid on PET or SPECT scans, and MRI in particular will provide complementary information on tissue biophysical and physiologic properties via, for example, assessments of tissue composition, water diffusion rates, or vascular properties. Moreover, some simplistic and conventional approaches to contrast enhancement in MRI are unlikely to be successful unless large concentrations of agent can be accumulated in tissue and the effects are

TABLE 6. Requirements of Microbubble Contrast Agents for Ultrasound

$\begin{array}{ccc}\begin{array}{c}\text { Radius of } \\ \text { particle }(\mathrm{nm})\end{array} & \begin{array}{c}\text { Particles per } \mathrm{mm}^{3} \\ \text { to be detected }\end{array} & \begin{array}{c}\text { Particles per cell } \\ \text { to be detected }\end{array} \\ 63,000 & 1 & 1 \text { every } 10^{6} \text { cells } \\ 6,300 & 10^{3} & 1 \text { every } 10^{3} \text { cells } \\ 630 & 10^{6} & 1 \text { per cell } \\ 100 & 25 \times 10^{7} & 250 \text { every cell } \\ 1,000 & 25 \times 10^{4} & 1 \text { every } 4 \text { cells } \\ 10,000 & 250 & 1 \text { every } 4,000 \text { cells }\end{array}$


not diluted by partial-volume averaging. It is the average relaxation rate within a voxel that must be changed for detection, and this rate needs to be high because the background rate is already significant. Direct visualization of nuclei such as fluorine will be possible when high concentrations can be achieved in small volumes, and hyperpolarization will dramatically increase the sensitivity for some materials. A general strategy that is more likely to be fruitful than small-molecule targeting is to engineer relatively large payloads, such as particles and liposomes, that may convey large numbers of a species to a target site. Our theoretic predictions suggest that even this approach may not be successful using small nanoparticles unless large numbers of them can be localized within a voxel, and that larger vehicles will be necessary. Moreover, similar strategies may also be potentially successful with CT, especially if conditions for imaging are changed to maximize sensitivity for molecular imaging at the expense of more conventional applications. Ultrasound bubbles that remain stable within the vasculature may have a role in detecting intravascular targets, and improvements in sensitivity may be expected using more advanced imaging methods than simple pulseecho backscattering, such as the detection of subharmonics and other nonlinear phenomena, but there seems little prospect of using much smaller bubbles than currently in use and considered here. We hope that these considerations will be useful for guiding further work in the development of multimodal contrast materials.

\section{ACKNOWLEDGMENTS}

Dr. Gore thanks the Society of Nuclear Medicine for the invitation to present the Henry Wagner, Jr., lecture at the annual meeting of the Society of Nuclear Medicine, Washington, DC, June 2007. This paper was presented in part during that lecture. Financial support for the Vanderbilt In Vivo Cellular and Molecular Imaging Center is provided by grant P50 CA128323 from the National Cancer Institute. Dr. Peterson is supported in part by a Career Award at the Scientific Interface from the Burroughs Wellcome Fund.

\section{REFERENCES}

1. Society of Nuclear Medicine. Molecular Imaging Center of Excellence Web site. Available at: http://www.molecularimagingcenter.org. Accessed October 20, 2008.

2. Mills PH, Ahrens ET. Theoretical MRI contrast model for exogenous T2 agents. Magn Reson Med. 2007;57:442-447.

3. Nunn AD, Linder KE, Tweedle MF. Can receptors be imaged with MRI agents? Q J Nucl Med. 1997;41:155-162.

4. Hanaoka K, Lubag AJ, Castillo-Muzquiz A, Kodadek T, Sherry AD. The detection limit of a Gd3+-based $\mathrm{T} 1$ agent is substantially reduced when targeted to a protein microdomain. Magn Reson Imaging. 2008;26:608-617.

5. Massoud TF, Gambhir SS. Molecular imaging in living subjects: seeing fundamental biological processes in a new light. Genes Dev. 2003;17:545-580.

6. Albert MS, Cates GD, Driehuys B, et al. Biological magnetic resonance imaging using laser-polarized ${ }^{129}$ Xe. Nature. 1994;370:199-201.

7. Black RD, Middleton HL, Cates GD, et al. In vivo He-3 MR images of guinea pig lungs. Radiology. 1996;199:867-870.
8. Strijkers GJ, Mulder WJ, van Tilborg GA, Nicolay K. MRI contrast agents: current status and future perspectives. Anticancer Agents Med Chem. 2007;7:291-305.

9. Sherry AD, Woods M. Chemical exchange saturation transfer contrast agents for magnetic resonance imaging. Annu Rev Biomed Eng. 2008;10:391-411.

10. Kreis R. Quantitative localized ${ }^{1} \mathrm{H}$ MR spectroscopy for clinical use. Prog Nucl Magn Reson Spectrosc. 1997;31:155-195.

11. Osorio JA, Ozturk-Isik E, Xu D, et al. 3D ${ }^{1} \mathrm{H}$ MRSI of brain tumors at 3.0 Tesla using an eight-channel phased-array head coil. J Magn Reson Imaging. 2007;26:23-30.

12. Golman K, Ardenkjaer-Larsen JH, Petersson JS, Mansson S, Leunbach I. Molecular imaging with endogenous substances. Proc Natl Acad Sci USA. 2003;100:10435-10439.

13. Chen AP, Albers MJ, Cunningham CH, et al. Hyperpolarized C-13 spectroscopic imaging of the TRAMP mouse at 3T-initial experience. Magn Reson Med. 2007;58:1099-1106.

14. Gabellieri C, Reynolds S, Lavie A, Payne GS, Leach MO, Eykyn TR. Therapeutic target metabolism observed using hyperpolarized $15 \mathrm{~N}$ choline. J Am Chem Soc. 2008;130:4598-4599.

15. Ishii M, Fischer MC, Emami K, et al. Hyperpolarized helium-3 MR imaging of pulmonary function. Radiol Clin North Am. 2005;43:235-246.

16. Albers MJ, Bok R, Chen AP, et al. Hyperpolarized ${ }^{13} \mathrm{C}$ lactate, pyruvate, and alanine: noninvasive biomarkers for prostate cancer detection and grading. Cancer Res. 2008;68:8607-8615.

17. Bhattacharya P, Chekmenev EY, Perman WH, et al. Towards hyperpolarized ${ }^{13} \mathrm{C}$ succinate imaging of brain cancer. J Magn Reson. 2007;186:150-155.

18. Bellin MF, Vasile M, Morel-Precetti S. Currently used non-specific extracellular MR contrast media. Eur Radiol. 2003;13:2688-2698.

19. Mohs AM, Lu ZR. Gadolinium(III)-based blood-pool contrast agents for magnetic resonance imaging: status and clinical potential. Expert Opin Drug Deliv. 2007;4:149-164.

20. Artemov D. Molecular magnetic resonance imaging with targeted contrast agents. J Cell Biochem. 2003;90:518-524.

21. Krishnan AS, Neves AA, de Backer MM, et al. Detection of cell death in tumors by using MR imaging and a gadolinium-based targeted contrast agent. Radiology. 2008;246:854-862.

22. Lepage M, Dow WC, Melchior M, et al. Noninvasive detection of matrix metalloproteinase activity in vivo using a novel magnetic resonance imaging contrast agent with a solubility switch. Mol Imaging. 2007;6:393-403.

23. Yoo B, Pagel MD. An overview of responsive MRI contrast agents for molecular imaging. Front Biosci. 2008;13:1733-1752.

24. Rogers WJ, Meyer $\mathrm{CH}$, Kramer CM. Technology insight: in vivo cell tracking by use of MRI. Nat Clin Pract Cardiovasc Med. 2006;3:554-562.

25. Bulte JW, Duncan ID, Frank JA. In vivo magnetic resonance tracking of magnetically labeled cells after transplantation. J Cereb Blood Flow Metab. 2002;22:899-907.

26. Gore JC, Joers JM, Kennan RP. Contrast agents and relaxation effects. In: Atlas $\mathrm{S}$, ed. Magnetic Resonance Imaging of the Brain and Spine. 3rd ed. Philadelphia, PA: Lippincott Williams and Wilkins; 2002:79-99.

27. Majumdar S, Zoghbi SS, Pope CF, Gore JC. A quantitative study of relaxation rate enhancement produced by iron-oxide particles in polyacrylamide gels and tissue. Magn Reson Med. 1989;9:185-202.

28. Zhang S, Merritt M, Woessner DE, Lenkinski RE, Sherry AD. PARACEST agents: modulating MRI contrast via water proton exchange. Acc Chem Res. 2003;36:783-790.

29. Woods M, Woessner DE, Sherry AD. Paramagnetic lanthanide complexes as PARACEST agents for medical imaging. Chem Soc Rev. 2006;35:500-511.

30. Morgan RH, Corrigan KE. Handbook of Radiology. Chicago, IL: Year Book Publishers; 1955.

31. Gore JC, Tofts PS. Statistical limitations in computed tomography. Phys Med Biol. 1978;23:1176-1182.

32. Swindell W, Webb S. X-ray transmission computed tomography. In: Webb S, ed. The Physics of Medical Imaging. Bristol, U.K.: Adam Hilger; 1988:123.

33. Hubbell JH, Seltzer SM. Tables of x-ray mass attenuation coefficients and mass energy-absorption coefficients. National Institute of Standards and Technology Web site, physical reference data. Available at: http://physics.nist.gov/PhysRefData/XrayMassCoef/cover.html. Accessed April 13, 2009.

34. Carroll F. Tunable, monochromatic X-rays: an enabling technology for molecular/cellular imaging and therapy. J Cell Biochem. 2003;90:502-508.

35. Sarnelli A, Elleaume H, Taibi A, Gambaccini M, Bravin A. K-edge digital subtraction imaging with dichromatic x-ray sources: SNR and dose studies. Phys Med Biol. 2006;51:4311-4328.

36. More P, Ingard K. Theoretical Acoustics. New York, NY: McGraw-Hill Book Co.; 1968.

37. Gore JC, Leeman S. Ultrasonic backscattering from human tissue: a realistic model. Phys Med Biol. 1977;22:317-326. 